\title{
Effects of Therapeutic Eccentric Exercise on Pain and Grip Strength in Persons with Lateral Epicondylitis: A Randomized Controlled Trial
}

\author{
${ }^{1}$ Nizar Abdul Majeedkutty, ${ }^{2}$ Nishad Abdul Latheef Majida \\ ${ }^{I}$ Senior Lecturer, Faculty of Medicine \& Health Sciences, University Tunku Abdul Rahman, Malaysia. \\ ${ }^{2}$ Senior Physiotherapist, A. M. Physiotherapy Centre, Kerala, India.
}

\begin{abstract}
Lateral epicondylitis produces a heavy burden of workdays lost and results in residual impairments. Though different treatment methods for lateral epicondylitis are available, majority of the studies reported inconsistent results and no therapeutic modality seems to stand out or alter the natural history of the disease. Eccentric exercise program increases the strength of the tendon by gradually overloading it to promote an increase in tensile strength. The purpose of this study was to investigate the effects of therapeutic eccentric exercises on pain and grip strength in subjects diagnosed with lateral epicondylitis. Twenty-four subjects (11 men and 13 women) with lateral epicondylitis were enrolled, randomized, and subdivided into 2 groups - an experimental group of 12 participants (55 \pm 2.12 years) and a control group of 12 participants with lateral epicondylitis $(53 \pm 2.27$ years $)$. They were evaluated at baseline and after 4 weeks of treatment. Control group received standard physical therapy and participants of the experimental group were subjected to therapeutic eccentric exercises along with standard physical therapy. Outcome measures included Visual Analogue Scale and Grip Strength. By the end of the trial period, the experimental group showed a significant improvement in scores of Visual Analogue Scale $(t=9.9469)$ and the grip strength $(t=26.90)$. The study showed that therapeutic eccentric exercise is more effective in reducing pain and improving grip strength in persons with lateral epicondylitis than standard physical therapy. The findings suggest that therapeutic eccentric exercise should be considered in the management of persons with lateral epicondylitis.
\end{abstract}

Keywords: lateral epicondylitis, pain, eccentric exercises, flexbar, grip strength

\section{Introduction}

Lateral epicondylitis (LE) is the most common overuse syndrome in general practice related to excessive wrist extension. Lateral epicondylitis is a painful disorder of the tendinous origin of the wrist extensor muscles [Piligian, 2000]. Although lateral epicondylitis is known as tennis elbow, the term is a misnomer as sufferers are more likely to be non-players. Any repetitive activity that places strain on the muscles of the forearm, particularly the outer muscles can cause small tears to develop in the tendon fibers. These tears occur at the point where the tendon attaches to the bone and occasionally in the covering of the bone. Diagnosis of epicondylitis is clinical and based on symptoms and findings of physical examination. The prevalence of lateral epicondylitis in the general population is approximately $1.0-1.3 \%$ in men and $1.1-4.0 \%$ in women [Shiri, 2011]. Although lateral epicondylitis occurs at all ages, the peak prevalence of lateral epicondylitis is between 30 and 60 years of age. The proportion of those afflicted by lateral epicondylitis is not influenced by the sex of the patient, but the disorder appears to be of longer duration and severity in females [Waugh, 2004]. LE is a degenerative or failed healing tendon response characterized by the increased presence of fibroblasts, vascular hyperplasia, and disorganized collagen in the origin of the extensor carpi radialis brevis (ECRB), the most commonly affected structure [Kraushaar, 1999].

LE is a relatively common condition that can affect persons who perform repetitive upper body activities such as carpenters, musicians, tennis players and computer programmers. From apathomechanical perspective, LE involves the proximal tendons of the extensor carpi radialis (ECR) and extensor digitorum (ED). The tendons are relatively hypovascular proximal to the tendon insertion.

This hypovascularity may predispose the tendon to hypoxic tendon degeneration and has been implicated in the etiology of tendinopathies. Most typically, the primary pathology in tendinosis of the extensor carpi radialis brevis (ECRB) tendon $1-2 \mathrm{~cm}$ dista to its attachment on the lateral epicondyle [Altan, 2008; Jafarian, 2009]. In general, microscopic evaluation of the tendons does not show signs of inflammation, but rather angiofibroblastic degeneration and collagen disarray. Light microscopy reveals both an excess of fibroblasts and blood vessels that are consistent with neo-vessels or angiogenesis [Bisset, 2006]. There has been a well-defined clinical presentation, the main complaints being pain and decreased grip strength. Diagnosis is simple and can be confirmed by the tests that reproduce pain such as palpation 
over the facet of the lateral epicondyle, resisted wrist extension, resisted middle finger extension and passive wrist flexion [Kamein, 1990].

A wide array of physiotherapy treatments have been recommended for the management of lateral epicondylitis [Stasinopoulos, 2005]. These treatments have different theoretical mechanisms of action, but all have the same aim, to reduce pain and improve function. Such a variety of treatment options suggests that the optimal treatment strategy is not known, and more research is needed to discover the most effective treatment in patients with lateral epicondylitis. Tendon injuries are difficult to manage, and current conservative and surgical management options have shown limited and often unpredictable success [Maffulli, 2008]. Interestingly, a systematic review and meta-analysis of interventions for LE noted a lack of evidence to support treatments other than exercise [Bisset, 2005]. Despite the prevalence of LE, only little consensus exists on its treatment [Bohr, 2011]. Though different treatment methods for lateral epicondylitis are available, majority of studies reported inconsistent results and no therapeutic modality seems to stand out or alter the natural history of the disease.

The concept of an eccentric exercise program is based on increasing the strength of the tendon by gradually overloading it to promote an increase in tensile strength. Furthermore, after performing an unaccustomed eccentric exercise and exhibiting soreness, the muscle quickly adapts and becomes accustomed to the increase in applied stress. As a result, not only is the soreness reduced, but other indicators of muscle damage are reduced. Muscles are designed to function eccentrically and to resist the mechanical strain placed upon them, as well as recover following an applied stress or strain. Until recently little was known of the effect of eccentric exercise on tendinopathies, although a greater understanding of this process is emerging. The best evidence to date does demonstrate that eccentric exercise is likely a useful management for tendinopathy, but this evidence is currently insufficient to suggest it is superior or inferior to other forms of therapeutic exercise [Rompe, 2007]. Therapeutic eccentric exercise (TEE) has been found to be an effective intervention for a variety of tendinopathies including Achilles tendinopathy, shoulder impingement, and patellar tendinopathy. Treatments for tendinopathy include rest, physical modalities (heat, electrical stimulation, ultrasound, and massage), drugs (oral and injection), stretching, and surgery. However, information regarding the effectiveness of these therapies is not conclusive. Moreover traditional treatment techniques are not directly addressing the issue of compromised tensile strength. It is suggested that eccentric exercise effectively "lengthened" the muscle-tendon complex resulting in structural remodeling of the tendon with hypertrophy and increased tensile strength of the tendon [Page, 2010]. Since these different types of tendinopathies show similarities in their histopathologic appearance, we decided to administer therapeutic eccentric training for lateral epicondylitis to provide pain relief and improve physical function. So this study was aimed to determine the effects of therapeutic eccentric exercises in the management of chronic lateral epicondylitis.

\section{Methods}

This randomized controlled prospective trial was conducted at A.M. Physiotherapy Centre in Kerala, India. The study was approved by the local ethics committee. A sample of 24 persons diagnosed with chronic lateral epicondylitis was recruited for the study. In this randomized study, 24 LE patients were assigned to either a control group $(n=12,5$ men, 7 women; age-53 \pm 2.27 years $)$ receiving standard physical therapy treatment or an experimental group $(n=12,6$ men, 6 women; age $-55 \pm 2.12$ years $)$ that received standard physical therapy with the addition of the therapeutic eccentric exercise using convenient sampling, with twelve subjects in each group. Patients diagnosed with lateral epicondylitis symptoms for greater than 4 weeks were enrolled for the study. Lateral epicondylitis was diagnosed using the following tests:

(1) Pain on palpation at the lateral epicondyle (2) Cozen's test (pain on resisted wrist extension) and (3) Maudsley's test (pain on resisted middle-finger extension). Subjects tested positive on all 3 tests were included in the study. Both male and female participants between the age of 30 and 60 years were included in the study. Patients with a history of fracture, dislocation, surgery, bilateral elbow pain, cervical spine pathology, osteoarthritis, or previous steroid injection to the elbow less than 6 weeks prior were excluded. The patients had no prior treatment for their lateral epicondylitis. We obtained informed consent from all participants. There was no significant difference between the groups prior to the intervention.

\section{Treatment protocol:}

All patients assigned to control group and experimental group received standard physical therapy which included ultrasound therapy and wrist extensor stretching during their physical therapy visits. All patients received pulsed ultrasonic therapy as standard physical therapy at $20 \%$ duty cycle, frequency $3 \mathrm{MHz}$ and an intensity of $1.2 \mathrm{~W} / \mathrm{cm} 2$ for 5 minutes, given at the teno-periosteal junction of the ECRB. The best stretching position for the Extensor Carpi Radialis Brevis tendon was reached with the elbow in extension, forearm in 
pronation, wrist in flexion and with ulnar deviation of the wrist, according to the patient's tolerance. This stretching was held for 15 seconds and 3 times during each treatment session with a 30 seconds rest interval. In addition to this, the experimental group performed therapeutic eccentric exercises. The isolated eccentric strengthening exercise was performed using a rubber bar (FlexBar; The Hygenic Corporation, Akron $\mathrm{OH}$ ) which was twisted using wrist flexion of the uninvolved limb and slowly allowed to untwist with eccentric wrist extension by the involved limb. Slow release was instructed for each eccentric wrist extensor contraction which lasted approximately 5 seconds. According to Selvier T; eccentric contractions should be performed at a slow velocity to avoid the possibility of re-injury [Selvier, 2000]. We chose Flexbar to provide therapeutic eccentric exercises because we thought it would make the experiment more reproducible. The authors believe that FlexBar would be a cost-effective, easy to use eccentric exercise method if it is found effective in the management of lateral epicondylitis. Once they could perform the exercises with little or no pain using the FlexBar, they were provided with FlexBar with increased resistance. The subjects performed 10 repetitions of exercise in one set. 3 sets of 10 repetitions were performed on three days a week for 4 weeks. It takes 4 seconds to complete each repetition and between each set of 10 repetitions there is 30 seconds of rest. However, they were instructed to stop the exercise if the pain becomes disabling. Each patient received written instructions with pictorial illustrations specific for treatment. They were told to avoid precipitating and exacerbating activities. Outcome Measurements:

The Visual Analog Scale (VAS) is a tool widely used to measure pain. The patient is asked to indicate his/her perceived pain intensity along a $100 \mathrm{~mm}$ horizontal line, and this rating is then measured from the left edge (VAS score). Pain is plotted along a line. The VAS is presented as a $10-\mathrm{cm}$ line, anchored by verbal descriptors, usually 'no pain' and 'worst imaginable pain'. VAS is valid, reliable and appropriate for use in clinical practice [Williamson, 2005]. The VAS and grip strength were assessed prior to and after the treatment period. The grip strength of the affected hand was measured using a standard adjustable handgrip dynamometer. We used the standardized procedure for positioning of instrument with subject seated with back, pelvis, and knees as close to 90 degrees as possible, shoulder is adducted and neutrally rotated, elbow flexed at 90 degrees, forearm neutral, wrist held between 0-15 degrees of ulnar deviation. The arm is not supported by examiner or armrest and the dynamometer is presented vertically and in line with the forearm [Horowitz, 1997]. Maximum grip is the mean of three trials. The use of the instrument to measure grip strength was illustrated to the participants prior to testing. Three readings were taken giving 30 seconds rest period in between to avoid fatigue. All outcome measures (VAS, Grip Strength) were assessed by trained physical therapists.

\section{Results}

The data was analyzed using statistical computer software SPSS 19 software package (SPSS Inc. Chicago, USA). Descriptive statistical analysis was carried out in the present study. The paired t-test and unpaired t-test was used. The level of significance was $p<0.05$. The two groups were found homogenous in terms of pain rated using visual analogue scale. There is no significant difference between mean ages between the groups. The pre-test VAS scores of control group and experimental group were compared using unpaired t test. The two-tailed $\mathrm{P}$ value equals 0.7584 . By conventional criteria, this difference is considered to be not statistically significant. $\quad(\mathrm{t}=0.3115, \mathrm{df}=22$, standard error of difference $=0.375)$. It was found that in both groups there was a statistically significant difference in VAS pain scores as compared by paired t-test. Improvement in VAS for pain was better for the eccentric group versus the standard treatment group. We used unpaired test to compare the post-test vas scores of both groups. The two-tailed $\mathrm{p}$ value equals 0.0455 . By conventional criteria, this difference is considered to be statistically significant. $(\mathrm{t}=2.1198, \mathrm{df}=22$, standard error of difference $=0.425)$. (Table: 1). Complete resolution of symptom was not found in any patient from both the groups. In the Eccentric exercise Group, 8 patients had $>25 \%$ improvement in VAS while in the Standard Treatment Group only 2 patients had $>25 \%$ improvement in VAS. It was shown that in both groups there was a statistically significant difference in grip strength scores as compared by paired t test (Table: 2). Improvement in grip strength was better for the eccentric group versus the standard treatment group.

\section{Discussion}

This study revealed that therapeutic eccentric exercises are effective in reducing pain and improving grip strength among persons with chronic lateral epicondylitis. Both outcome measures for chronic lateral epicondylitis were markedly improved with the addition of therapeutic eccentric exercise to standard physical therapy. Analysis of the results in both the groups showed a statistically significant improvement in VAS score and Grip strength. Subjects in the experimental group procured more substantial benefits in reducing pain and improving grip strength by adding therapeutic eccentric exercises to standard physical therapy. Classically, pain in tendinopathy has been attributed to inflammatory processes, but, as it has become evident that tendinopathies are not inflammatory conditions, that they present histological evidence of a failed healing response and that even the degenerative paradigm is not really applicable, recently the 
combination of mechanical and biochemical causes has become more attractive. Microscopic tendon disruption with mechanical breakdown of collagen could theoretically explain the pain mechanism, but clinical and surgical observations challenge this view. Substance P and chondroitin sulphate may also be involved in producing pain in tendinopathy [Khan, 2000]. Also, the decrease in pain may be a result from a lengthening of the musculo-tendinous junction creating less strain and allowing a greater range of motion. The biochemical model has become appealing, as many chemical irritants and neurotransmitters may generate pain in tendinopathy. High concentrations of the neurotransmitter glutamate have been found in patients with Achilles tendinopathy. The tendons in these patients showed no signs of inflammation, as indicated by the normal prostaglandin E2 levels [Alfredson, 1999]. Another possible mechanism for pain reduction is neovascularization. Tendinosis pain is also associated with neovascularization but the pain may stem from the mechanical stimulation of pressure-sensitive autonomic nerves in the muscular walls of the arteries [Pufe, 2003]. According to Ohberg L et al eccentric exercise may halt the growth of blood vessels in tendinosis and subsequently relieve some of the associated pain [21].

The results of our study are in accordance with the findings of a randomized controlled trial conducted by Roos EM et al in 2004 [22]. He and his colleagues found that eccentric exercise is more effective in treating tendinosis than splinting. A similar study was conducted among persons with chronic patellar tendinopathy in which eccentric exercises were compared with some physical agents [Stasinopoulos, 2004]. Anyway the location of the threshold between safe and unsafe eccentric loading is unclear. Physical therapists must be aware of the continuum of factors that affect tendinous adaptation and ensure that the adopted protocol optimizes healing without producing harmful stresses. With respect to eccentric training for chronic lateral epicondylitis, Croisier et al compared isokinetic eccentric wrist extensor training to standard physical therapy [Croisier, 2007]. The effects of eccentric training on pain scores were very similar to the present study. However it is uncertain that how exercise program relieves pain. It is claimed that eccentric training results in tendon strengthening by stimulating mechanoreceptors in tenocytes to produce collagen, which is probably the key cellular mechanism that determines recovery from tendon injuries. In addition, eccentric training may induce a response that normalizes the high concentrations of glycosaminoglycan. It may also improve collagen alignment of the tendon and stimulate collagen crosslinkage formation, both of which improve tensile strength [Khan, 2000].

According to Hampson $\mathrm{K}$ et al mechanical conditioning can be used as a treatment for enhancing tendon healing. Mechano-transduction is the process of a cell converting mechanical stimuli into biochemical signals [Hampson, 2008]. Cells able to sense the mechanical signals are described as being mechanosensitive. Tendon responds to mechanical forces by adapting its metabolism and structural and mechanical properties. Tendons adapt to alterations in the mechanical load being applied by changing their structure and composition. The tenocytes in the tendon are responsible for its adaptive response, and respond to mechanical forces by altering their gene expression patterns, protein synthesis and cell phenotype, which can be used to aid the healing process. It has also been proposed that the positive effects of exercise program for tendon injuries may be attributable to either the effect of stretching, with a lengthening of the muscle-tendon unit and consequently less strain experienced during joint motion, or the effects of loading within the muscle-tendon unit, with hypertrophy and increased tensile strength in the tendon [Alfredson, 1998]. They speculated that since the energy pathways in the tendon become more anaerobic (without oxygen) as we age, the tendon might need to adapt to these anaerobic demands to avoid injury. A study from Sweden suggested that eccentric training and static stretching for 12 weeks can reduce pain and increase grip strength in lateral epicondylitis [Svernlov, 2011].

The theory behind eccentric strengthening is to load the musculotendinous unit inducing hypertrophy and increasing tensile strength. This in turn reduces the strain on the tendon during activities. Eccentric contraction can create a greater stimulus for the cells of the tendon, producing collagen and resulting in the tendon being able to withstand greater forces. Decreasing neovascularization has been recently documented as another benefit of eccentric strengthening. It is believed that neovascularization is a causing factor of pain in LE and other tendionpathies [Calfee, 2008]. Eccentric execution results in greater force production with less energy expenditure and less oxygen consumption compared to concentric execution. Nosaka et al. demonstrated the 'repeated bout effect'. After full recovery has been achieved following the first eccentric overload bout, a repeated training results in minimal symptoms of muscle damage allowing eccentric overload to become a viable training means, especially when considered that the 'repeated bout effect' can last for several months. The exact mechanisms are not well defined but it seems to involve neural, mechanical and cellular adaptations [Nosaka, 2001]. Therapeutic exercise programs appear to reduce pain and improve function in persons with lateral epicondylitis. Current literature has found connections between eccentric loading and positive outcomes in tendonopathy patients. Once a muscle fatigues the tendon accepts the kinetic forces hence the reason tendonopathy injuries are observed in overuse repetitive type athletes. Main components comprising tendinopathy are angiofibroblastic hyperplasia 
which is an internal misalignment of collagen fibers. Each time the degenerative tendon is further worked it restarts the fibroblastic phase of healing laying down new Type III collagen further degenerate the components of a normal tendon. Another reason for positive outcomes could be the simple increase in tensile strength due to loading induced hypertrophy [Rees, 2009]. Recent evidence suggests that eccentric actions may be more effective, but must be used with caution due to the common effect of muscle soreness. Traditional treatment techniques were not directly addressing this issue of compromised tensile strength. Progressively overloading the tendon overtime through eccentric exercises would then lead to an increase in tensile strength [Stanish, 2000]. Moreover this treatment can be performed as part of a home program and does not involve continued medical supervision. In the light of these facts, we can conclude that the effect of therapeutic eccentric exercise training on stimulating tendon remodeling and producing muscular adaptive responses has led to reduction in pain and improvement in grip strength in persons with lateral epicondylitis.

\section{Limitations and Recommendations}

A small sample size is a limitation of this study. Investigators in future trials should recruit larger numbers of subjects. No long term follow- up data was collected past 4 weeks; therefore the long-term effects of the interventions in the present study remain unknown. Future research is warranted that would determine the long term effects of the interventions used in this study. We also recommend physical therapists to follow the eccentric exercise protocol for optimal reduction of symptoms among persons with lateral epicondylitis.

\section{Conclusion}

It was concluded that persons with lateral epicondylitis procured more substantial benefits in reducing pain and improving grip strength by adding therapeutic eccentric exercises to standard physical therapy. The findings suggest that therapeutic eccentric exercise should be considered in the management of persons with lateral epicondylitis.

\section{References}

[1]. Alfredson H, Pietila T, Johnson P (1998). Heavy-load eccentric calf muscle training for the treatment of chronic Achilles tendinosis. American Journal of Sports Medicine, 26:360-6.

[2]. Alfredson H, Thorsen K, Lorentzon R (1999). In situ microdialysis in tendon tissue: high levels of glutamate, but not prostaglandin E2 in chronic Achilles tendon pain. Knee Surgery, Sports Traumatology, Arthroscopy, 7:378-81.

[3]. Altan L, Kanat E (2008). Conservative treatment of lateral epicondylitis: comparison of two different orthotic devices. Clinical Rheumatology, 27(8):1015-9.

[4]. Bisset L, Beller E, Jull G, Brooks P, Darnell R, Vicenzino B (2006). Mobilisation with movement and exercise, corticosteroid injection, or wait and see for tennis elbow: randomised trial. British Medical Journal, 333(7575): 939.

[5]. Bisset L, Paungmali A, Vicenzino B, Beller E (2005). A systematic review and meta-analysis of clinical trials on physical interventions for lateral epicondylalgia. British Journal of Sports Medicine, 39(7): 411-422.

[6]. Bohr PC (2011). Systematic review and analysis of work-related injuries to and conditions of the elbow. American Journal of Occupational Therapy, 65(1):24-28.

[7]. Calfee RP, Patel A, DaSilva MF, Akelman E (2008). Management of lateral epicondylitis: Current concepts. Journal of American Academy of Orthopaedic Surgery, 16(1):19-29.

[8]. Croisier JL, Foidart-Dessalle M, Tinant F, Crielaard JM, Forthomme B (2007). An isokinetic eccentric programme for the management of chronic lateral epicondylar tendinopathy. British Journal of Sports Medicine, 41:269-75. doi:10.1136/bjsm.2006.033324

[9]. Hampson K, Forsyth NR, El Haj A, Maffulli N (2008). Tendon tissue engineering. Topics in tissue engineering, 4; 1-21.

[10]. Horowitz BP, Tollin R \& Cassidy G (1997). Grip strength: Collection of normative data with community dwelling elders. Physical and Occupational Therapy in Geriatrics, 15: 53-64.

[11]. Jafarian FS, Demneh ES, Tyson SF (2009). The immediate effect of orthotic management on grip strength of patients with lateral epicondylosis. Journal of Orthopaedic \& Sports Physical Therapy, 39(6): 484-9.

[12]. Kamein M (1990). A rational management of tennis elbow. Sports Medicine, 9: 173-91.

[13]. Khan KM, Cook JL, Maffulli N, Kannus P (2000). Where is the pain coming from in tendinopathy? It may be biochemical, not only structural, in origin. British Journal of Sports Medicine, 34:81-3.

[14]. Khan K, Cook J, Taunton J (2000). Overuse tendinosis, not tendinitis: a new paradigm for a difficult clinical problem. The Physician and Sports medicine, 28:38-48.

[15]. Kraushaar B, Nirschl R (1999). Current concepts review - tendinosis of the elbow (tennis elbow). Clinical features and findings of histological, immunohistochemical and electron microscopy studies. Journal of Bone \& Joint Surgery, 81:259-85.

[16]. Maffulli N, Longo UG (2008). Conservative management for tendinopathy: is there enough scientific evidence? Rheumatology, 47:390-1.

[17]. Nosaka K, Sakamoto K, Newton M, \& Sacco P (2001). The repeated bout effect of reduced-load eccentric exercise on elbow flexor muscle damage. European Journal of Applied Physiology, 85: 34-40.

[18]. Ohberg L, Alfredson H (2004). Effects on neovascularisation behind the good results with eccentric training in chronic mid-portion Achilles tendinosis? Knee Surgery, Sports Traumatology, Arthroscopy, 12:465-470.

[19]. Page P (2010). A new exercise for tennis elbow that works. North American Journal of Sports Physical Therapy, 5(3): 189-193. PMCID: PMC2971639.

[20]. Piligian G, Herbert R, Hearns M, Dropkin J, Landsbergis P, Cherniack M (2000). Evaluation and management of chronic workrelated musculoskeletal disorders of the distal upper extremity. American Journal of Industrial Medicine, 37(1): 75-93. 
[21]. Pufe T, Petersen W, Kurz B, Tsokos M, Tillmann B, Mentlein R (2003). Mechanical factors influence the expression of endostatin, an inhibitor of angiogenesis in tendons. Journal of Orthopaedic Research, 21:610-616.

[22]. Rees JD, Wolman RL, Wilson A (2009). Eccentric exercises; why do they work, what are the problems and how can we improve them? British Journal of Sports Medicine, 43(4):242-246.

[23]. Roos EM, Engstrom M, Lagerquist A, Soderberg B (2004). Clinical improvement after 6 weeks of eccentric exercise in patients with mid-portion Achilles tendinopathy: a randomized trial with 1-year follow-up. Scandinavian Journal of Medicine \& Science in Sports, 14:286-295.

[24]. Rompe JD, Nafe B, Furia JP, Maffulli N (2007). Eccentric loading, shock-wave treatment, or a wait-and-see policy for tendinopathy of the main body of Tendo-Achillis: a randomized controlled trial. American Journal of Sports Medicine, 35:374-83

[25]. Selvier T, Wilson J (2000). Methods utilized in treating lateral epicondylitis. Physical Therapy Reviews, 5:117-24.

[26]. Shiri R, Juntura E (2011). Lateral and medial epicondylitis: Role of occupational factors. Best Practice \& Research Clinical Rheumatology, 25: 43-57.

[27]. Stasinopoulos D, Johnson MI (2005). Effectiveness of extracorporeal shock wave therapy for tennis elbow: a review. British Journal of Sports Medicine; 39:132-6.

[28]. Stasinopoulos D, Stasinopoulos I (2004). Comparison of effects of exercise programme, pulsed ultrasound and transverse friction in the treatment of chronic patellar tendinopathy. Clinical Rehabilitation, 18: 347-352.

[29]. Stanish WD, Curwin S, Mandell S (2000). Tendinitis: its etiology and treatment. New York, Oxford University Press.

[30]. Svernlov B, Adolfsson L (2011). Non-operative treatment regime including eccentric training for lateral humeral epicondylalgia. Scandinavian Journal of Medicine and Science in Sports, 11: 328-334.

[31]. Waugh E, Jaglal S, Davis A, et al. (2004). Factors associated with prognosis of lateral epicondylitis after 8 weeks of physical therapy. Archives of Physical Medicine and Rehabilitation, 85:308-18.

[32]. Williamson A, Hoggart B (2005). Pain: a review of three commonly used pain rating scales. Journal of Clinical Nursing, 14, 798804.

\begin{tabular}{|c|c|c|c|c|}
\hline VAS & Pre-test & Post-test & t value & Standard Error of \\
\hline Standard Treatment Group I & $6.77 \pm 0.924$ & $5.62 \pm 0.975$ & $\mathrm{t}=4.0186$ & 0.286 \\
\hline Eccentric Exercise Group II & $6.65 \pm 0.911$ & $4.72 \pm 1.10$ & $\mathrm{t}=9.9469$ & 0.194 \\
\hline
\end{tabular}

Table: 1 Comparing pre-test and post-test values of VAS Score for both groups

\begin{tabular}{|l|c|c|c|}
\hline \multicolumn{1}{|c|}{ Grip strength } & Pretest & Post test & t-value \\
\hline Standard Treatment Group I & $14.12 \pm 0.890$ & $17.613 \pm 1.224$ & 14.39 \\
\hline Eccentric Exercise Group II & $14.00 \pm 1.192$ & $18.80 \pm 1.102$ & 26.90 \\
\hline
\end{tabular}

Table: 2 Comparing pre-test and post-test values of Grip strength Score for both groups 